\title{
Minimizing Differential Modal Gain in Cladding Pumped MM-EDFAs for Mode Division Multiplexing in $C$ and $L$ Bands
}

\author{
Qiongyue Kang,* Ee-Leong Lim, Francesco Poletti, Yongmin Jung, Shaif-ul Alam, and David J. Richardson \\ Optoelectronics Research Centre, University of Southampton, Southampton, SO17 1BJ, UK \\ "qk1g11@orc.soton.ac.uk
}

\begin{abstract}
We propose the cladding pumped four-mode-group and six-mode-group EDFA designs for mode division multiplexing transmission systems that provide less than $1 \mathrm{~dB}$ Differential Modal Gain (DMG) across the wavelength range of 1530-1600nm.

OCIS codes: (060.2320) Fiber optics amplifiers and oscillator; (060.2410) Fibers, erbium.
\end{abstract}

\section{Introduction}

Space Division Multiplexing (SDM) has attracted considerable attention in high-capacity fiber-optic communication systems as a radical approach to increase the capacity per-fiber [1]. One form of SDM uses Few Mode Fibers (FMFs) which guide a restricted number of modes to define the independent spatial channels. So far, both 2 and 4mode group systems have been reported, with inline Few-Mode Erbium Doped Fiber Amplifier (FM-EDFA) [2-4]. The majority of the demonstrated FM-EDFAs have been core-pumped with either one or two single-mode pump diodes [4-5]. Obviously, as the number of modes supported by a single fiber is increased, the total signal output power (and hence the required pump power needed for high gain, low noise amplification) increases significantly, dictating the use of multiple, expensive single mode pumps. Reduction in the cost per transmitted bit is essential for SDM to be seriously considered for commercial deployment and cladding-pumping using high-power multimode pump diodes (which has already been demonstrated for multi-core EDFAs [6]) provides a more practical and potentially much cheaper way to provide the pump radiation. The first demonstration of cladding pumped EDFA supporting four mode groups reported a DMG (i.e. the maximum gain difference among all the signal modes at the same wavelength) of around $4 \mathrm{~dB}$ [7]. When developing FM EDFAs, minimizing DMG is of paramount importance in ensuring the best overall system performance. In the modeling of cladding-pumped amplifiers, a simple but effective way to simulate the pump is to assume that the pump intensity profile is uniform across the doped core [89]. So careful tailoring of the erbium doping profile in an active multimode fiber is therefore central to minimizing the DMG in cladding-pumped FM-EDFAs.

In this work, we employ a Genetic Algorithm (GA) [10] to help optimize the rare earth doping profile of a cladding-pumped EDFA, supporting more than two propagating mode groups, in order to reduce the DMG. The optimum fiber designs provide less than $1 \mathrm{~dB}$ DMG across the wavelength range (1530-1600nm).

\section{Simulation}

The FM-EDFA was simulated using the simulation model described in our previous work [11]. For the noise calculation, we split the wavelength band from $1490 \mathrm{~nm}$ to $1630 \mathrm{~nm}$ into 140 equal-width $(1 \mathrm{~nm})$ wavelength slots. In order to manipulate the erbium ion distribution, we chose to divide the erbium-doped core into several layers whilst keeping the FRIP unchanged, so that the optimal dopant concentration in each core layer can be numerically investigated for minimization of the DMG. For the purpose of reducing fabrication complexity, the number of erbium-doped layers should be as few as possible. Through trials, we have found that at least three-layer and a fourlayer doping structures (shown in Fig. 1) are required in order to achieve a DMG of less than $1 \mathrm{~dB}$ in the C- band $(1530-1565 \mathrm{~nm})$ for the 4M-EDFA and 6M-EDFA respectively. Thus for the 4M-EDFA, there are two structural parameters (i.e. $a_{1}, a_{2}$ shown in Fig. 1a) determining the dimensions of the three-core layers and three doping concentration parameters (i.e. $\rho_{1}, \rho_{2}, \rho_{3}$ ) describing the erbium ion concentration of each core layer to be optimized. Similarly, there are three structural parameters (i.e. $a_{1}, a_{2}, a_{3}$ shown in Fig. 1b) and four doping concentration parameters (i.e. $\rho_{1}, \rho_{2}, \rho_{3}, \rho_{4}$ ) to be optimized for the 6M-EDFA. Due to the involvement of more than 5 free parameters, we implemented a GA to establish the optimum choice of the dopant concentration and the dimension of each core layer for the minimization of the DMG. The GA is a generic optimization tool for optimizing multiple parameters simultaneously based on a natural selection process that mimics biological evolution. The GA has been successfully applied to several fiber designs, for example ref. [12]. In the GA implemented here, the fitness function, "F", that is used to evaluate the 'quality' of a given structure and minimized by the algorithm, is defined as:

$$
F=\sum_{\lambda_{i}=1530 \mathrm{~nm}}^{1565 \mathrm{~nm}} D M G\left(\lambda_{i}\right)
$$


where $\operatorname{DMG}\left(\lambda_{i}\right)$ is the differential modal gain calculated at wavelength $\lambda_{i}$ and the sum is performed over 5 uniformly spaced points in the interval. For all modeling work in this paper, a total input signal power of $0 \mathrm{dBm}$ at a single wavelength, co-propagating with a pump input power of $2.5 \mathrm{~W}$ are assumed. It is to be noted here that the total input signal power is equally split into the 6 spatially distinct signal modes in the case of $4 \mathrm{M}$-EDFA (i.e. $\mathrm{LP}_{01}$, $\mathrm{LP}_{11 \mathrm{a}}$, $\mathrm{LP}_{11 \mathrm{~b}}, \mathrm{LP}_{21 \mathrm{a}}, \mathrm{LP}_{21 \mathrm{~b}}$ and $\mathrm{LP}_{02}$ ), i.e. $-7.78 \mathrm{dBm}$ per mode; and 10 spatially distinct signal modes in the case of the $6 \mathrm{M}-$ EDFA (i.e. $\mathrm{LP}_{01}, \mathrm{LP}_{11 \mathrm{a}}, \mathrm{LP}_{11 \mathrm{~b}}, \mathrm{LP}_{21 \mathrm{a}}, \mathrm{LP}_{21 \mathrm{~b}}, \mathrm{LP}_{02}, \mathrm{LP}_{31 \mathrm{a}}, \mathrm{LP}_{31 \mathrm{~b}}, \mathrm{LP}_{12 \mathrm{a}}, \mathrm{LP}_{12 \mathrm{~b}}$ ), i.e. $-10 \mathrm{dBm}$ per mode. The amplifier model is run five times (to obtain the DMG values needed at different signal wavelengths) to calculate the fitness function ' $F$ ' for a given fiber structure. The free parameters (or the 'genes' of the algorithm) are the structural parameters $a_{i}\left(i . e . a_{1}, a_{2}, \ldots\right)$ and the doping concentration $\rho_{i}\left(i . e . \rho_{1}, \rho_{2}, \ldots\right)$ of the $i$-th core layer.

\section{Optimized fiber}

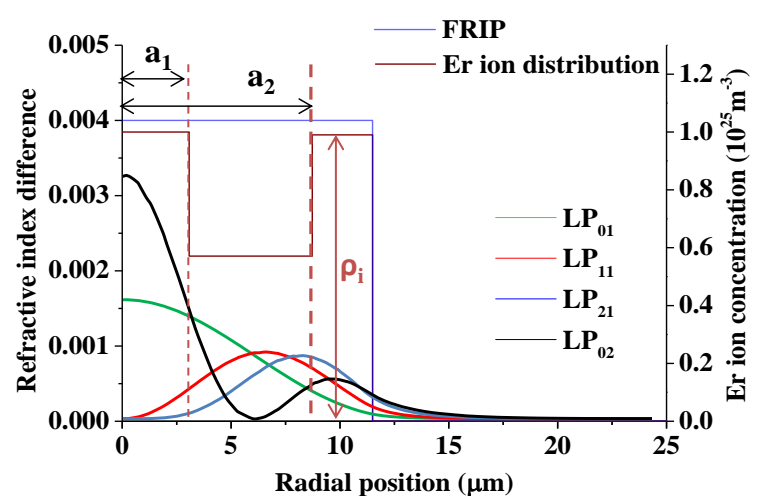

(a)

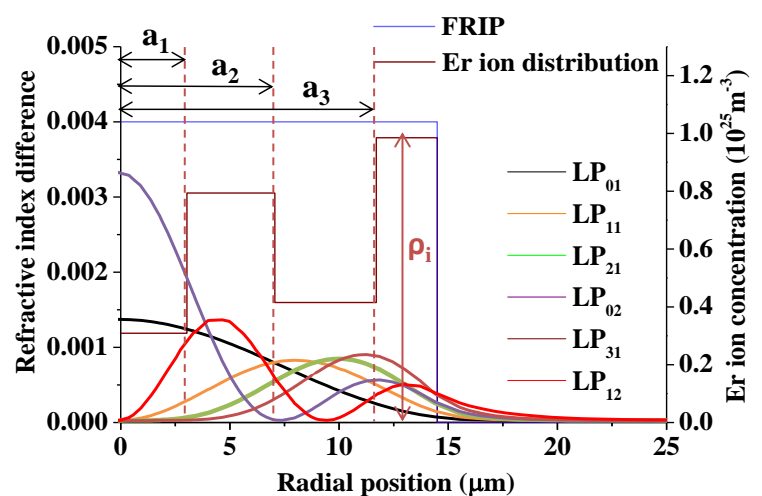

(b)

Figure 1. The fiber refractive index profile (FRIP), the signal mode intensity distributions, and the doping profile of (a) 4M-EDFA, denoted as 'F1' and (b) 6M- EDFA, denoted as 'F2', to be optimized through the GA; where $\rho_{\mathrm{i}}\left(\mathrm{m}^{-3}\right)$ is the doping concentration of the $\mathrm{i}$-th core layer.

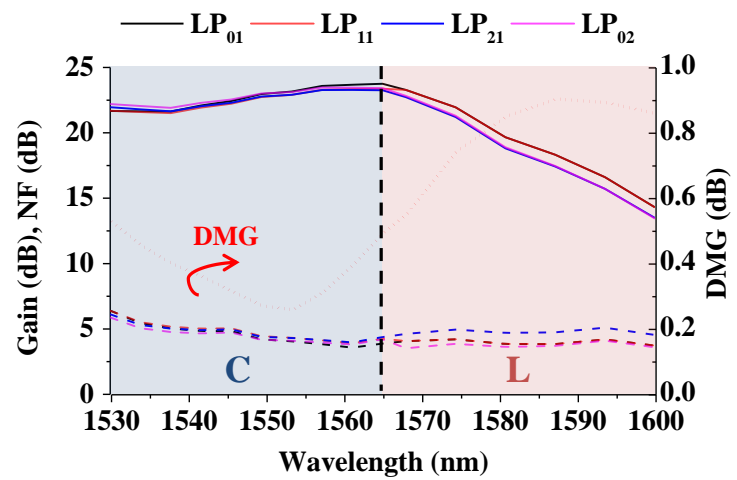

(a)

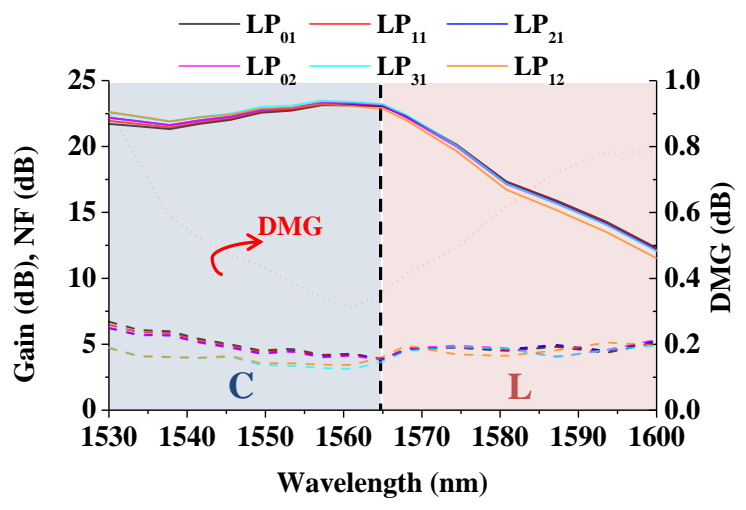

(b)

Figure 2. The modal gain (continuous line), noise figure (dashed line) and DMG (red dotted line) characteristics of the best (a) 4-mode-group EDFA and (b) 6-mode-group EDFA calculated by the GA. The blue (pink) shaded region indicates the C-band (L-band).

\section{(1) Four-mode-group EDFA}

We have chosen to study a double-clad, step-index erbium doped fiber (EDF) with a core-to- inner clad refractive index difference of 0.004 and a core diameter of $23 \mu \mathrm{m}$. We assume that the fiber is weakly guiding and supports the lowest four transverse mode groups from $1530 \mathrm{~nm}$ to $1565 \mathrm{~nm}$, whose modal intensity profiles are shown in Fig. 1a. The diameter of the inner cladding was chosen to be $100 \mu \mathrm{m}$, compatible with our preferred choice of pigtailed pump diode, and results in a core-to-cladding ratio of about 1: 4.3. The best fiber design $\left(\mathrm{a}_{1}=3.074 \mu \mathrm{m}, \mathrm{a}_{2}=8.730 \mu \mathrm{m}, \rho_{1}\right.$ $=1.00 \mathrm{e}+25 \mathrm{~m}^{-3}, \rho_{2}=5.71 \mathrm{e}+24 \mathrm{~m}^{-3}, \rho_{3}=9.98 \mathrm{e}+24 \mathrm{~m}^{-3}$ ) found through the GA is shown in Fig. 1a, here denoted as 'F1'. This ' $\mathrm{W}$ ' shaped erbium dopant distribution provides similar amounts of overlap between the dopants and the signal modes. The gain and noise characteristics of the best fiber design are plotted in Fig. 2a. A fiber length of $13 \mathrm{~m}$ is used to ensure minimal gain tilt across the $\mathrm{C}$-band, as indicated by the blue-shaded region in Fig. 2a. The gain and 
noise properties in the L-band is also plotted in the pink-shaded region as shown in Fig. 2a. The DMG (shown in red dotted line in Fig. 2a) is controlled to better than $1 \mathrm{~dB}$ from $1530 \mathrm{~nm}$ to $1600 \mathrm{~nm}$, with a minimum of $0.26 \mathrm{~dB}$ at $1553 \mathrm{~nm}$ and maximum of $0.90 \mathrm{~dB}$ at $1587 \mathrm{~nm}$. In the $\mathrm{C}$ band, the modal gains are found to be more than $20 \mathrm{~dB}$ with a maximum gain of $23.37 \mathrm{~dB}$ for the $\mathrm{LP}_{01}$ mode at around $1565 \mathrm{~nm}$ and a minimal gain of $21.51 \mathrm{~dB}$ for the LP 11 mode at around $1538 \mathrm{~nm}$, for the input signal/pump power conditions described in section 2. The NFs shown in Fig. $2 \mathrm{a}$ are relatively high (i.e. between 5 to $7 \mathrm{~dB}$ ) at the short wavelength region $(1530-1542 \mathrm{~nm})$ where the gain is relatively low. When the gain is relatively high, in the wavelength region of $1545 \mathrm{~nm}-1565 \mathrm{~nm}$, the NFs are found to be below $5 \mathrm{~dB}$. In the L-band, the modal gains are much lower than those in the C-band, because the amplifier length is not optimized to achieve high gain in the L-band.

(2) Six-mode-group EDFA

In this instance the core diameter of the EDF is scaled up to $29 \mu \mathrm{m}$ so as to support six-mode-groups while keeping the core-to-inner clad refractive index difference and the inner cladding diameter exactly the same as in the previous case. The FRIP and signal intensity profiles of the 6M-EDFA (denoted as 'F2') and its optimal erbium dopant profile $\left(a_{1}=3.030 \mu \mathrm{m}, a_{2}=7.059 \mu \mathrm{m}, a_{3}=11.703 \mu \mathrm{m}, \rho_{1}=3.09 \mathrm{e}+24 \mathrm{~m}^{-3}, \rho_{2}=7.94 \mathrm{e}+24 \mathrm{~m}^{-3}, \rho_{3}=4.15 \mathrm{e}+24 \mathrm{~m}^{-3}, \rho_{4}=\right.$ $9.85 \mathrm{e}+24 \mathrm{~m}^{-3}$ ) obtained from the GA are shown in Fig. 1b. A fiber length of $12 \mathrm{~m}$ is used to ensure minimal gain tilt across the $\mathrm{C}$ band, as shown in the blue-shaded region in Fig. $2 \mathrm{~b}$. The gain and noise properties in the L-band are also plotted in the pink-shaded region in Fig. 2b. As shown in Fig. 2b, the DMGs (the red dotted line) are found to be below $1 \mathrm{~dB}$ with a lowest value of $0.31 \mathrm{~dB}$ at $1561 \mathrm{~nm}$ and a highest value of $0.91 \mathrm{~dB}$ at $1530 \mathrm{~nm}$. The gain and noise characteristics of F2 are similar to that of F1. In the C-band, the modal gains are well above $20 \mathrm{~dB}$ with a maximum of $23.47 \mathrm{~dB}$ for the $\mathrm{LP}_{31}$ mode at around $1557 \mathrm{~nm}$ and a minimum of $21.38 \mathrm{~dB}$ for the $\mathrm{LP}_{01}$ mode at around $1538 \mathrm{~nm}$. The NFs of the $\mathrm{LP}_{01}, \mathrm{LP}_{11}$ and $\mathrm{LP}_{21}$ modes are relatively high (larger than $5 \mathrm{~dB}$ ) at the short wavelength region (i.e. $1530-1540 \mathrm{~nm}$ ) but again become less than $5 \mathrm{~dB}$ for wavelengths longer than $1545 \mathrm{~nm}$. Again, the modal gains in the L-band are not optimum. However, the optimization of gains in the L-band is beyond the scope of this paper.

From more extensive simulations, we have also found that the changes in length or pump powers (in order to optimize gain, NF at certain signal wavelengths) of the amplifiers based on fibers F1 or F2 in general only result in a very small variation of the DMGs (less than 5\%).

\section{Conclusion}

We applied a Genetic Algorithm to minimize the differential modal gain in cladding pumped EDFAs supporting four and six-mode-groups. The optimum 4M-EDFA and 6M-EDFA designs, exhibiting three-layer and four-layer core structures (denoted as 'F1' and 'F2) respectively, provide less than $1 \mathrm{~dB}$ DMG across the C- and L-band. Over $20 \mathrm{~dB}$ gain across the $\mathrm{C}$-band is obtained for both F1 and F2 using a forward pump power of 2.5W and EDF lengths of $13 \mathrm{~m}$ and $12 \mathrm{~m}$ respectively. The amplifier performance in the L-band can be further improved by optimizing the fiber length and pump power.

\section{References}

[1] D. J. Richardson, et al. , "Space-division multiplexing in optical fibers", Nat. Photonics 7, 354-362 (2013).

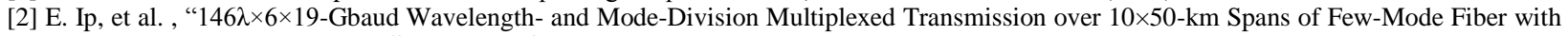
a Gain-Equalized Few-Mode EDFA", Proc. OFC'13, Paper PDP5A.2 (2013).

[3] V. A. J. M. Sleiffer, et al. , "73.7 Tb/s (96 x 3 x 256-Gb/s) mode-division-multiplexed DP-16QAM transmission with inline MM-EDFA", Opt. Express 20(26), B428-B438 (2012)

[4] M. Salsi, et al. , "A six mode erbium-doped fiber amplifier", Proc. ECOC' 12, Paper Th.3.A (2012).

[5] Y. Jung, et al., "Few-mode EDFA supporting 5 spatial modes with reconfigurable differential modal gain control", Proc. ECOC'13, Paper We.4.A.2 (2013).

[6] K. S. Abedin, et al., "Cladding pumped erbium doped multicore fiber amplifier", Opt. Express 20(18), 20191-20200 (2012).

[7] E. Lim, et al. , "First Demonstration of Cladding Pumped Few-moded EDFA for Mode Division Multiplexed Transmission", Proc. OFC'14, Paper M2J.2 (2014)

[8] D. J. Richardson, et al., "High power fiber lasers: current status and future perspectives," J. Opt. Soc. Am. B: Opt. Phys. 27, B63-B92 (2010).

[9] A. Hardy, "Signal amplification in strongly pumped fiber amplifiers," IEEE J. of Quantum Electron. 33, 307-313 (1997).

[10] D.E. Goldberg, Genetic algorithms in search, optimization and machine learning, (Addison-Wesley, New York, 1989).

[11] Q. Kang, et al., "Accurate modal gain control in a multimode erbium doped fiber amplifier incorporating ring doping and a simple $\mathrm{LP}_{01}$ pump configuration ”, Opt. Express 20 (19), 20835-20843 (2012).

[12] F. Poletti, et al. , "Inverse design and fabrication tolerances of ultra-flattened dispersion holey fibers", Opt. Express 13(10), 3728-3736 (2005).

\section{Acknowledgement}

This work was supported by the European Communities 7th Framework Program under grant agreement 258033 (MODE-GAP). 